\title{
GS100-02-41: a new large $H$ । shell in the outer part of the Galaxy
}

\author{
L. A. Suad ${ }^{1}$, S. Cichowolski ${ }^{2}$, E. M. Arnal ${ }^{1,3}$, and J. C. Testori ${ }^{1}$
}

\author{
${ }^{1}$ Instituto Argentino de Radioastronomía (IAR) (CCT - La PLata), CC 5, 1894 Villa Elisa, Argentina \\ e-mail: lasuad@iar.unlp.edu.ar \\ 2 Instituto de Astronomía y Física del Espacio (IAFE), CC 67, Suc. 28, 1428 Buenos Aires, Argentina \\ ${ }^{3}$ Facultad de Ciencias Astronómicas y Geofísicas, Universidad Nacional de La Plata, Argentina
}

Received 13 September 2011 / Accepted 26 November 2011

\section{ABSTRACT}

\begin{abstract}
Context. Massive stars have a profound effect on the surrounding interstellar medium. They ionize and heat the neutral gas, and with their strong winds they sweep up the gas, forming large Hi shells. In this way, they generate a dense shell that provides the physical conditions for the formation of new stars.

Aims. The aim of this study is to analyze the origin and evolution of the large Hi shell GS 100-02-41 and its role in triggering star-forming processes.

Methods. To characterize the shell and its environs, we carried out a multi-wavelength study. We analyzed the $\mathrm{H}_{\text {I }} 21 \mathrm{~cm}$ line, the radio continuum, and infrared emission distributions.

Results. The analysis of the $\mathrm{H}_{\mathrm{I}}$ data shows an expanding shell structure centered at $(l, b)=\left(100^{\circ} .6,-2^{\circ} .04\right)$ in the velocity range from -29 to $-51.7 \mathrm{~km} \mathrm{~s}^{-1}$. Taking into account noncircular motions, we infer for GS 100-02-41 a kinematical distance of $2.8 \pm 0.6 \mathrm{kpc}$. Several massive stars belonging to Cep OB1 are located in projection within the large $\mathrm{H}_{\mathrm{I}}$ shell boundaries. The analysis of the radio continuum and infrared data reveals that there is no continuum counterpart of the Hi shell. On the other hand, three slightly extended radio continuum sources are observed in projection onto the dense H i shell. From their flux density determinations we infer that they are thermal in nature. An analysis of the $\mathrm{H}_{\mathrm{I}}$ emission distribution in the environs of these sources shows a region of low emissivity for each of them, which correlates well morphologically with the ionized gas in a velocity range similar to the one where GS 100-02-41 is detected.

Conclusions. Based on an energy analysis, we conclude that the origin of GS 100-02-41 could have been mainly caused by the action of the Cep OB1 massive stars located inside the $\mathrm{H}_{\text {I }}$ shell. The obtained age difference between the $\mathrm{H}_{\mathrm{I}}$ shell and the $\mathrm{H}_{\text {II }}$ regions, together with their relative location, lead us to conclude that the ionizing stars could have been created as a consequence of the shell evolution.
\end{abstract}

Key words. ISM: structure - ISM: kinematics and dynamics - HII regions - stars: formation

\section{Introduction}

Giant structures with linear dimension of a few hundred parsecs in diameter are widely known and well-observed phenomena in the interstellar medium (ISM) of the Milky Way, when viewed in the $\lambda \sim 21 \mathrm{~cm}$ line emission of neutral hydrogen (HI). They were first noticed by Heiles (1979). These structures are usually detected as huge shells or arc-like features of enhanced $\mathrm{H}_{\mathrm{I}}$ emission that surrounds regions of low $\mathrm{H}_{\mathrm{I}}$ emissivity, the so-called H I supershells. These features may even be the dominant structure in the interstellar medium because they make up a large part of the volume of the galactic disk. The $\mathrm{H}_{\mathrm{I}}$ structures can also be observed at infrared wavelengths. Based on the 60 and $100 \mu \mathrm{m}$ IRAS databases, Könyves et al. (2007) have performed an allsky survey of loop- and arc-like structures.

Similar structures have also been observed in nearby spiral galaxies (Stanimirović 2007; Chakraborti \& Ray 2011). In the Milky Way, these structures were initially cataloged by Heiles (1979, 1984). Though a large number of $\mathrm{H}_{\mathrm{I}}$ features that are likely to be classified as either large $\mathrm{HI}_{\mathrm{I}}$ shells or $\mathrm{HI}_{\mathrm{I}}$ supershells have been cataloged in the outer $\left(90^{\circ} \leq l \leq 270^{\circ}\right)$ part of the Galaxy (Ehlerová \& Palouš 2005), only a small number of them have been studied in any detail. These (Jung et al. 1996; Stil \& Irwin 2001; Uyaniker \& Kothes 2002; McClure-Griffiths et al. 2002; Cazzolato \& Pineault 2003; Arnal \& Corti 2007; Cichowolski \& Pineault 2011) have galactocentric distances ranging from 9.7 to $16.6 \mathrm{kpc}$, diameters from 120 to $\sim 840 \mathrm{pc}$, expansion velocities between $\sim 10$ and $\sim 20 \mathrm{~km} \mathrm{~s}^{-1}$, and kinetic energies from $\sim 1 \times 10^{50}$ up to $\sim 6 \times 10^{51} \mathrm{erg}$. Among them only two (GS 305+01-24 and the feature studied by Cazzolato \& Pineault 2003) have an OB-association as their likely powering source, and another three (GSH 91.5+2-114, GS 234-02 and GS 263$02+45)$ show evidence of having induced the formation of a new generation of stars.

The general consensus is that those structures whose kinetic energy is on the order of, or less than, a few times $10^{51}$ erg, very likely may have been created by the joint action of stellar winds and supernova explosions. Very many examples are reported in the literature (e.g. Uyaniker \& Kothes 2002; Arnal \& Corti 2007; Cichowolski \& Pineault 2011). On the other hand, for expanding $\mathrm{H}_{\mathrm{I}}$ structures with kinetic energies in excess $\sim 10^{52} \mathrm{erg}$, termed supershells, the above mechanism may not be adequate to create them because one would need a stellar grouping (either an open cluster or an OB-association) with many more stars than the average found in the Milky Way. In these cases alternative mechanisms such as the infalling of high-velocity clouds (TenorioTagle 1981) or gamma-ray bursts (Perna \& Raymond 2000) may be at work.

Along its expansion, these structures (either a shell or a supershell) may become gravitationally unstable, forming clouds that consequently may lead to the formation of stars along the periphery of these $\mathrm{H}$ I structures, or else the expanding structure 
may hit and compress pre-existing ISM molecular clouds from one side. During this process a high-density perturbation may move into the molecular clouds, which may eventually collapse into denser cores in which star formation may occur. A thorough review of observations and theory related to the triggering of star formation is given by Elmegreen (1998).

A new large-scale study aiming at detecting structures in the outer part of the Galaxy that are likely to be either large $\mathrm{H}_{\mathrm{I}}$ shells or supershells is being carried out by one of the authors (Suad) as part of her Ph.D. Thesis.

In this paper we analyze a new large $\mathrm{H}_{\mathrm{I}}$ shell observed at $(l, b) \sim\left(100^{\circ},-2^{\circ}\right)$, with the purpose of elucidating both its origin and its interaction with the surrounding ISM. We also look for signs of recent star-formation activity that is likely to be related to this shell.

\section{Observations}

Low-resolution $\mathrm{H}_{\mathrm{I}}$ data were retrieved from the LeidenArgentine-Bonn (LAB) survey (Kalberla et al. 2005). This database is well-suited for a study of large-scale structures because of its angular resolution. The entire database has been corrected for stray radiation. High angular resolution $\mathrm{H}_{\mathrm{I}}$ data, covering most of the structure under study, were obtained from the Canadian Galactic Plane Survey (CGPS, Taylor et al. 2003). Besides the high-resolution $\mathrm{H}_{\mathrm{I}}$ data, the CGPS also provides high-resolution continuum data at 408 and $1420 \mathrm{MHz}$ (Landecker et al. 2000). Continuum data at $2695 \mathrm{MHz}$ (Reich et al. 1984, 1990; Fürst et al. 1990) were also used in this study.

Infrared data from the Midcourse Space Experiment (MSX) (Price et al. 2001) were obtained from the Infrared Science Archive $^{1}$. Infrared images from the Improved Reprocessing of the IRAS Survey (IRIS) (Miville-Deschênes \& Lagache 2005) were retrieved from the SkyView homepage ${ }^{2}$.

Carbon monoxide data $\left({ }^{12} \mathrm{CO}(1 \rightarrow 0)\right)$ for a small area centered at $(l, b) \sim(100.7,-0.5)$ were kindly made available to us by Dr. Christ Brunt, while for $l \geq 102.08{ }^{12} \mathrm{CO}$ data were retrieved from the CGPS database. These data were obtained using the 14-m dish of the Five College Radio Astronomy Observatory (FCRAO).

Table 1 summarizes the most relevant observational parameters.

The use of this multi-wavelength approach allows us to probe the different components (neutral and ionized gas and dust) of the ISM.

\section{GS $100-02-41$}

\subsection{Neutral hydrogen data}

We consider that a given $\mathrm{H}_{\mathrm{I}}$ structure may be classified as a shell if the following criteria are fullfilled:

1. it must have a well-defined lower brightness temperature surrounded (partially or completely) by regions of higher temperature;

2. the Hi minimum must be observable in at least five consecutive velocity channels;

\footnotetext{
1 The NASA/IPAC Infrared Science Archive is operated by the Jet Propulsion Laboratory, California Institute of Technology, under contract with the Nasa Aeronautics and Space Administration

(http://irsa.ipac.caltech.edu).

${ }^{2}$ http://skyview.gsfc.nasa.gov/
}

Table 1. Observational parameters.

\begin{tabular}{lc}
\hline \hline LAB H I data & $34^{\prime}$ \\
Angular resolution & $1.3 \mathrm{~km} \mathrm{~s}^{-1}$ \\
Velocity resolution & -450 to $400 \mathrm{~km} \mathrm{~s}^{-1}$ \\
Velocity coverage & \\
CGPS H I data & $1.2 \times 1^{\prime}$ \\
Angular resolution & $1.3 \mathrm{~km} \mathrm{~s}^{-1}$ \\
Velocity resolution & -164 to $58 \mathrm{~km} \mathrm{~s}^{-1}$ \\
Velocity coverage & \\
Radio continuum & $3.5 \times 4^{\prime} .6$ \\
Angular resolution (408 MHz) & $1.2 \times 1^{\prime}$ \\
Angular resolution (1420 MHz) & $4 !^{\prime}$ \\
Angular resolution (2695 MHz) & \\
CO data & $\sim 1^{\prime}$ \\
Angular resolution & $0.824 \mathrm{~km} \mathrm{~s}^{-1}$ \\
Velocity resolution & $18^{\prime \prime} 4$ \\
Infrared data & $3 !^{\prime} .4 \mathbf{4}^{\prime} .3$ \\
Angular resolution (MSX) & $0.5-2^{\prime}$ \\
Angular resolution (IRIS) & \\
Angular resolution (HIRES) & \\
\hline
\end{tabular}

3. it must have a minimum angular size of $2^{\circ}$. This condition is set bearing in mind the angular resolution of the LAB survey, to ensure that the structure under study is, angularly speaking, fully resolved;

4. at the kinematic distance of the structure, its linear size must exceed $200 \mathrm{pc}$.

Relying on the criteria described above, we are constructing a large $\mathrm{H}_{\mathrm{I}}$ shells and supershells catalog in the outer part of the Galaxy (Suad et al., in prep.). As part of this catalog, we have found an $\mathrm{H}_{\mathrm{I}}$ structure located at $(l, b, V)=\left(100^{\circ} .6,-2^{\circ} .3\right.$, $\left.-41 \mathrm{~km} \mathrm{~s}^{-1}\right)$. Following the standard nomenclature, this shell is labeled GS 100-02-41.

To illustrate the main observational findings, in Fig. 1 a mosaic of six $\mathrm{H}_{\mathrm{I}}$ images covering the velocity range form -47.41 to $-31.96 \mathrm{~km} \mathrm{~s}^{-1}$ is shown (all velocities in this paper are given with reference to the LSR). Each image is an average of three individual line channel maps and covers a velocity interval of about $3.09 \mathrm{~km} \mathrm{~s}^{-1}$.

The upper left panel shows the $\mathrm{H}_{\mathrm{I}}$ brightness temperature distribution at $-47.41 \mathrm{~km} \mathrm{~s}^{-1}$. In addition to the $\mathrm{HI}_{\mathrm{I}}$ emission arising from the galactic plane, quite a few regions of low emissivity are clearly identified. These regions appear as a local minimum surrounded along most of its perimeter by regions of higher brightness temperature, such as the structure located at $(l, b)=\left(101^{\circ} 0,-2^{\circ} .0\right)$. As we move toward more positive velocities, this structure becomes a well-defined $\mathrm{H}_{\mathrm{I}}$ minimum within the overall galactic $\mathrm{H}_{\mathrm{I}}$ emission. This minimum achieves its maximum angular extent at $-41.2 \mathrm{~km} \mathrm{~s}^{-1}$ and is barely visible at $-32 \mathrm{~km} \mathrm{~s}^{-1}$ (lower right panel).

An average of the $\mathrm{H}_{\mathrm{I}}$ emission distribution in the velocity range from -42.26 to $-34.02 \mathrm{~km} \mathrm{~s}^{-1}$ is shown in Fig. 2, where a huge $\mathrm{H}_{\mathrm{I}}$ structure with dimensions of $\sim 6^{\circ} \times 3^{\circ}(\Delta l \times \Delta b)$ is easily recognizable.

To estimate the main parameters of this large $\mathrm{H}_{\mathrm{I}}$ shell, we have characterized the ellipse that best fits it using a leastsquares method. The points used to fit the ellipse correspond to the local maxima around the cavity. To select these points the CGPS H I data were used. The parameters derived from this fit are the symmetry center of the ellipsoidal $\mathrm{H}_{\mathrm{I}}$ distribution $\left(l_{0}\right.$, $\left.b_{0}\right)$, the length of both the semi-major $(a)$ and semi-minor $(b)$ axes of the ellipse, and the inclination angle $(\theta)$ between the 

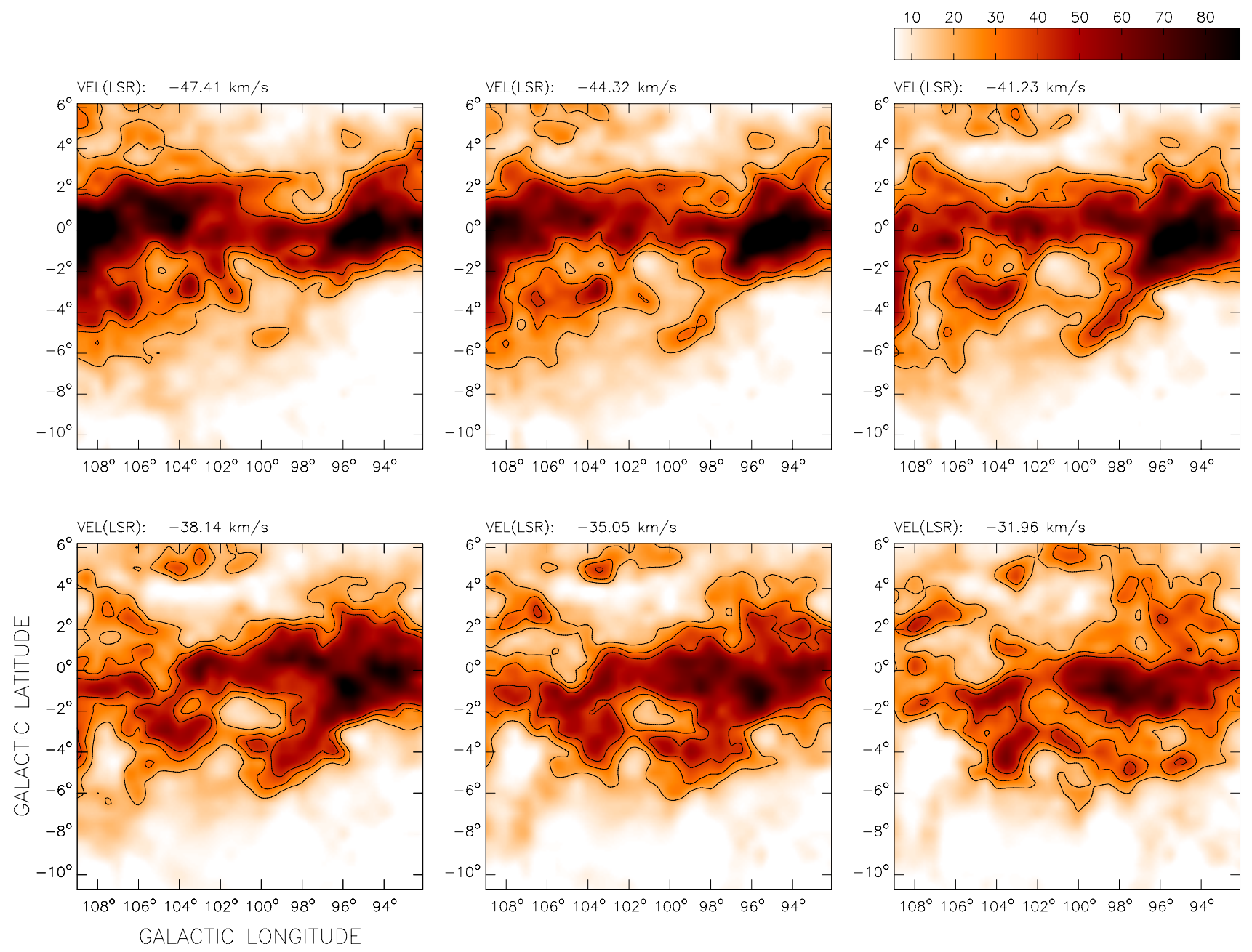

Fig. 1. LAB Hi emission distribution in the velocity range from -47.41 to $-31.96 \mathrm{~km} \mathrm{~s}^{-1}$. Each image is an average of three velocity channel maps. Contour levels are at 20,30, and $40 \mathrm{~K}$. The LSR central velocity of each image is indicated in its top left corner. The color bar-code shows the temperature scale in kelvin for all the images.

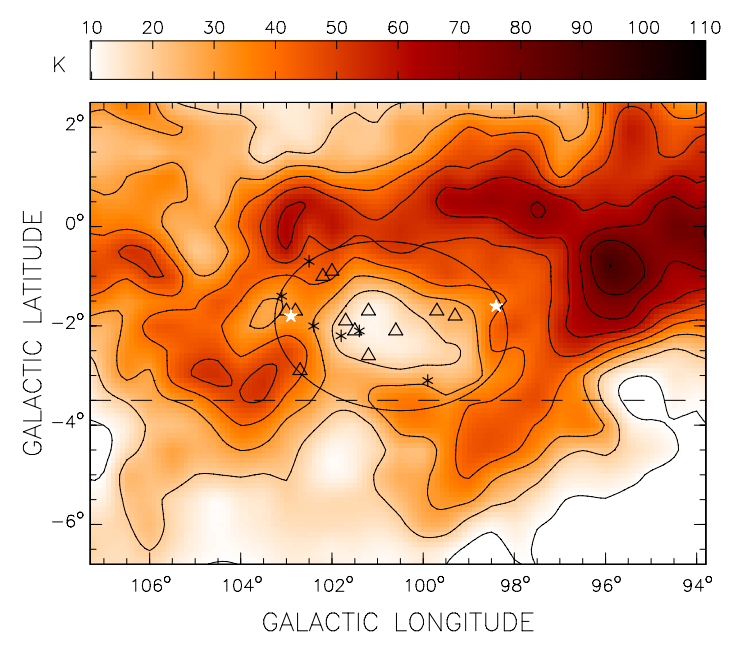

Fig. 2. $\mathrm{LAB} \mathrm{H}_{\mathrm{I}}$ emission distribution averaged in the velocity range from -42.26 to $-34.02 \mathrm{~km} \mathrm{~s}^{-1}$. Contour levels are from 10 to 90 in steps of $10 \mathrm{~K}$. The area below the dashed line corresponds to the region where CGPS data are not available. The symbols indicate the location of the star members of Cep OB1 lying inside the ellipse (see Sect. 3.3). White star symbols correspond to O-type main-sequence stars. Asterisks and triangles indicate evolved stars that were O-type and B-type stars during the main-sequence phase, respectively.
Table 2. GS 100-02-41 main parameters.

\begin{tabular}{lc}
\hline \hline Parameter & Value \\
\hline Distance $(\mathrm{kpc})$ & $2.8 \pm 0.6$ \\
$\left(l_{0}, b_{0}\right)$ & $(100.6,-2.04)$ \\
$a$ & 2.56 \\
$b$ & 1.69 \\
$\theta$ & $-5^{\circ} .7$ \\
$a(\mathrm{pc})$ & $125 \pm 25$ \\
$b(\mathrm{pc})$ & $83 \pm 17$ \\
$V_{0}\left(\mathrm{~km} \mathrm{~s}^{-1}\right)$ & $-41 \pm 2$ \\
$V_{\mathrm{e}}\left(\mathrm{km} \mathrm{s}^{-1}\right)$ & $11 \pm 2$ \\
$M_{\mathrm{t}}\left(M_{\odot}\right)$ & $(1.5 \pm 0.7) \times 10^{5}$ \\
$n_{\mathrm{sh}}\left(\mathrm{cm}^{-3}\right)$ & $2.5 \pm 0.4$ \\
$n_{0}\left(\mathrm{~cm}^{-3}\right)$ & $1.7 \pm 0.4$ \\
$E_{\mathrm{k}}\left(\mathrm{erg}^{\circ}\right)$ & $(1.8 \pm 0.8) \times 10^{50}$ \\
$t_{\mathrm{dyn}}(\alpha=0.25)(\mathrm{Myr})$ & $2.3 \pm 1.1$ \\
$t_{\mathrm{dyn}}(\alpha=0.6)(\mathrm{Myr})$ & $5.5 \pm 2.7$ \\
\hline
\end{tabular}

major axis and the galactic longitude axis. This angle is positive toward the north Galactic pole. They are given in Table 2.

Under the assumption of a symmetric expansion, an ellipsoidal $H_{\text {I }}$ feature with a central velocity $V_{0}$ and an expansion velocity $V_{\mathrm{e}}$ should depict, in a position-position diagram, an ellipse-like pattern when observed at different radial velocities. 


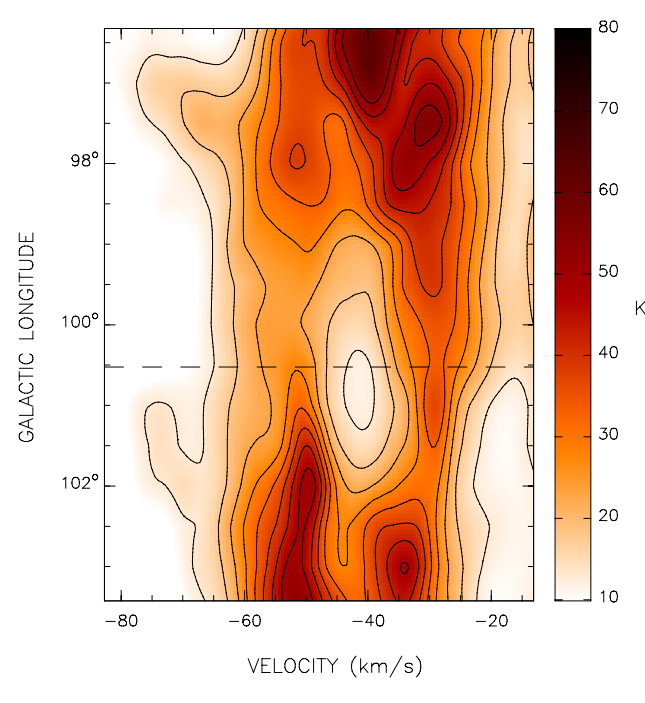

Fig. 3. $\mathrm{LAB} \mathrm{H}_{\mathrm{I}}$ emission distribution averaged in the latitude range from $-3^{\circ} .0$ to -1.3 . Contour levels are from 13 to $53 \mathrm{~K}$ in steps of $5 \mathrm{~K}$. The dashed line indicates the location where the cross-cut shown in Fig. 4 was performed.

At $V_{0}$ the ellipse of $\mathrm{H}_{\mathrm{I}}$ emission attains its maximum dimensions, while at extreme velocities (either approaching $\left(V_{\mathrm{m}}=\right.$ $\left.V_{0}-V_{\mathrm{e}}\right)$ or receding $\left.\left(V_{\mathrm{M}}=V_{0}+V_{\mathrm{e}}\right)\right)$ the hydrogen emission should look like an "ovoidal" patch of emission. At intermediate velocities the dimension of the $\mathrm{H}_{\mathrm{I}}$ ellipse shrinks as $V_{\mathrm{M}}\left(\right.$ or $\left.V_{\mathrm{m}}\right)$ is approached. The expansion velocity is estimated to be half of the total velocity range $\left(V_{\mathrm{e}}=0.5\left(\left|V_{\mathrm{M}}-V_{\mathrm{m}}\right|\right)\right)$ covered by the $\mathrm{H}_{\mathrm{I}}$ emission related to the feature. This method always provides a lower limit to $V_{\mathrm{e}}$, because $\mathrm{H}_{\mathrm{I}}$ emission arising from those regions with radial velocities close to either the maximum approaching $\left(V_{\mathrm{m}}\right)$ or receding $\left(V_{\mathrm{M}}\right)$ cap are usually difficult to distinguish from the overall galactic $\mathrm{H}_{\mathrm{I}}$ emission. Another way to determine $V_{\mathrm{e}}$ without the above drawback is to use the velocity-position diagrams. In Fig. 3 a radial velocity versus galactic longitude diagram is shown. This image shows a different view of GS 100-02-41. The inner region of the elliptical Hi feature shown in Fig. 2 corresponds to the low-emissivity region seen at $(V, l)=\left(-41 \mathrm{~km} \mathrm{~s}^{-1}, 100^{\circ} 6\right)$. The strong peaks of $\mathrm{HI}_{\mathrm{I}}$ emission seen at $\sim-29 \mathrm{~km} \mathrm{~s}^{-1}$ and $\sim-51 \mathrm{~km} \mathrm{~s}^{-1}$ arise from the $\mathrm{H}_{\mathrm{I}}$ associated with the expanding walls of neutral gas related to GS 100-02-41. Figure 4 shows a cross-cut of Fig. 3 along $l=100^{\circ}$. . By making a Gaussian fit to the $\mathrm{H}_{\mathrm{I}}$ emission peaks, $V_{\mathrm{m}}$ and $V_{\mathrm{M}}$ can be derived, along with the value of $V_{0}$, that corresponds to the velocity between $V_{\mathrm{m}}$ and $V_{\mathrm{M}}$ where the minimum value of $T_{b}$ is observed. We obtain $V_{0}=-41 \pm 2 \mathrm{~km} \mathrm{~s}^{-1}$ and $V_{\mathrm{e}}=11 \pm 2 \mathrm{~km} \mathrm{~s}^{-1}$.

It is well known that non-circular motions on a large scale are present in the Perseus spiral arm (Brand \& Blitz 1993), making the galactic rotation model not suitable for this region of the Galaxy. Based on the observed velocity field derived by Brand \& Blitz (1993), the systemic velocity of GS 100-02-41 can be translated into a kinematic distance of $2.8 \pm 0.6 \mathrm{kpc}$. We adopted this distance for the H I shell.

Under the assumption that the $\mathrm{H}_{\mathrm{I}}$ emission is optically thin and following the procedure described by Pineault (1998), the total neutral hydrogen mass of a structure located at a distance $d$ (kpc) that subtends a solid angle $\Omega$ (square arc-min) is given by

$M_{\mathrm{HI}}\left(M_{\odot}\right)=1.3 \times 10^{-3} d^{2} \Delta V \Delta T_{\mathrm{B}} \Omega$,

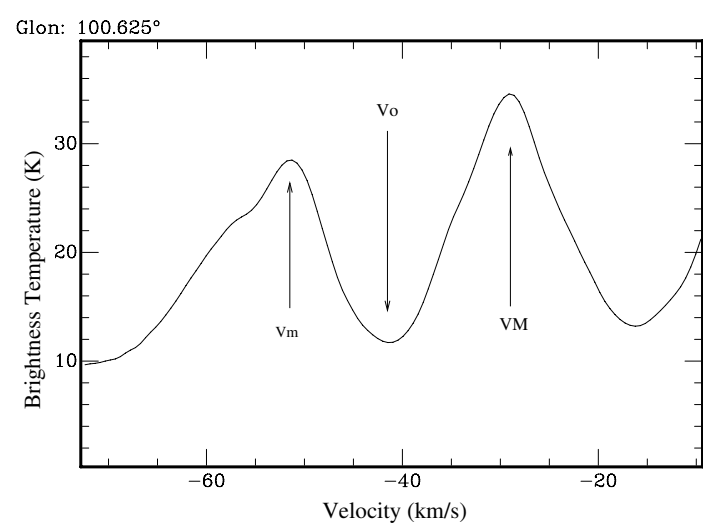

Fig. 4. H i cross-cut at $l=1000^{\circ} 6$ obtained from Fig. 3 . The approaching $\left(V_{\mathrm{m}}\right)$, systemic $\left(V_{0}\right)$ and receding $\left(V_{\mathrm{M}}\right)$ velocities are indicated.

where $\Delta V$ is the velocity interval over which the structure is detected, expressed in $\mathrm{km} \mathrm{s}^{-1}$ and $\Delta T_{\mathrm{B}}(\mathrm{K})$ is the mean brightness temperature defined as $\Delta T_{\mathrm{B}}=\left|T_{\mathrm{sh}}-T_{\mathrm{bg}}\right|$, where $T_{\mathrm{sh}}$ refers to the mean brightness temperature of the $\mathrm{H}_{\mathrm{I}}$ shell, and $T_{\mathrm{bg}}$ corresponds to the temperature of the contour level defining the outer border of the $\mathrm{H}_{\mathrm{I}}$ shell. The latter represents the temperature of the surrounding galactic $\mathrm{H}_{\mathrm{I}}$ emission gas. For this structure we estimated $T_{\mathrm{sh}}=33.5 \pm 1.6 \mathrm{~K}, T_{\mathrm{bg}}=21.5 \pm 1.5 \mathrm{~K}$, $\Delta V=22 \pm 2 \mathrm{~km} \mathrm{~s}^{-1}$ and $\Omega=4.2 \times 10^{4} \operatorname{arcmin}^{2}$. Adopting solar abundances, the total gaseous mass of GS 100-02-41 is $M_{\mathrm{t}}\left(M_{\odot}\right)=1.34 M_{\mathrm{HI}}$ (see Table 2$)$.

Assuming that the mass is uniformly distributed within the structure's volume, we obtain the gas number density $\left(n_{\mathrm{sh}}\right)$ of the swept up gas. The ambient density $\left(n_{0}\right)$ of the medium into which the large $\mathrm{H}_{\mathrm{I}}$ shell is evolving is derived by uniformly distributing the shell mass $\left(M_{\mathrm{t}}\right)$ over the volume swept up by the structure. Assuming this mass to be distributed into an ovoidal volume whose semi-major axis is $a$ and the other two dimensions are set equal to $b$,

$n_{0}=10 \frac{M_{\mathrm{t}}}{a b^{2}}\left(\mathrm{~cm}^{-3}\right)$,

where $M_{\mathrm{t}}$ is given in $M_{\odot}$, and $a$ and $b$ in parsecs.

A rough estimate of the age of the shell can be obtained using a simple model to describe the expansion of a shell created by a continuous injection of mechanical energy or by a supernova explosion. In this way, the dynamical age of GS 100-02-41 can be estimated as $t_{\mathrm{dyn}}=\alpha R / V_{\mathrm{e}}$ (Weaver et al. 1977), where $\mathrm{R}$ is the radius of the shell $(R=\sqrt{a b})$, and $\alpha=0.25$ for a radiative supernova remnant (SNR) or $\alpha=0.6$ for a stellar wind shell.

Another important parameter that characterizes the shells is the kinetic energy, which is given by $E_{\mathrm{k}}=0.5 M_{\mathrm{t}} V_{\mathrm{e}}^{2}$. All relevant parameters of GS 100-02-41 are given in Table 2.

Even though the CGPS data do not cover the whole area of GS 100-02-41, for completeness we show in Fig. 5 the CGPS data averaged in the same velocity range as the LAB data. A comparison with Fig. 2 clearly shows that the large-scale structures are observed in both data sets and, as expected, a lot of small-scale structures are observed in the high-resolution image. The dashed ellipse shown in Fig. 5 is the one obtained form the fitting of the low-resolution data (see Table 2).

\subsection{Radio continuum and infrared data}

Figure 6 shows the observed radio continuum emission at $2695 \mathrm{MHz}$ (upper panel) and the $60 \mu \mathrm{m}$ infrared emission (lower 


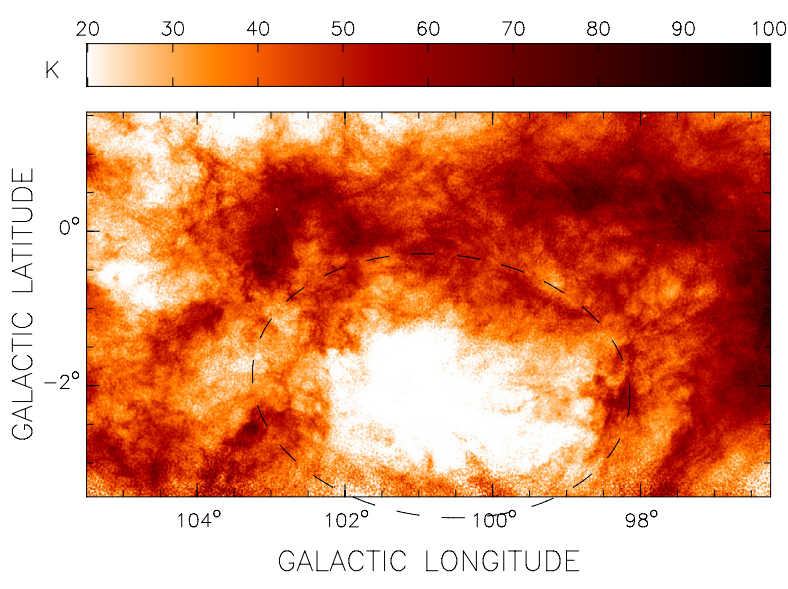

Fig. 5. CGPS averaged $\mathrm{H}_{\mathrm{I}}$ emission distribution in the velocity range from -42.7 to $-34.4 \mathrm{~km} \mathrm{~s}^{-1}$. The dashed ellipse corresponds to the ellipse shown in Fig. 2.

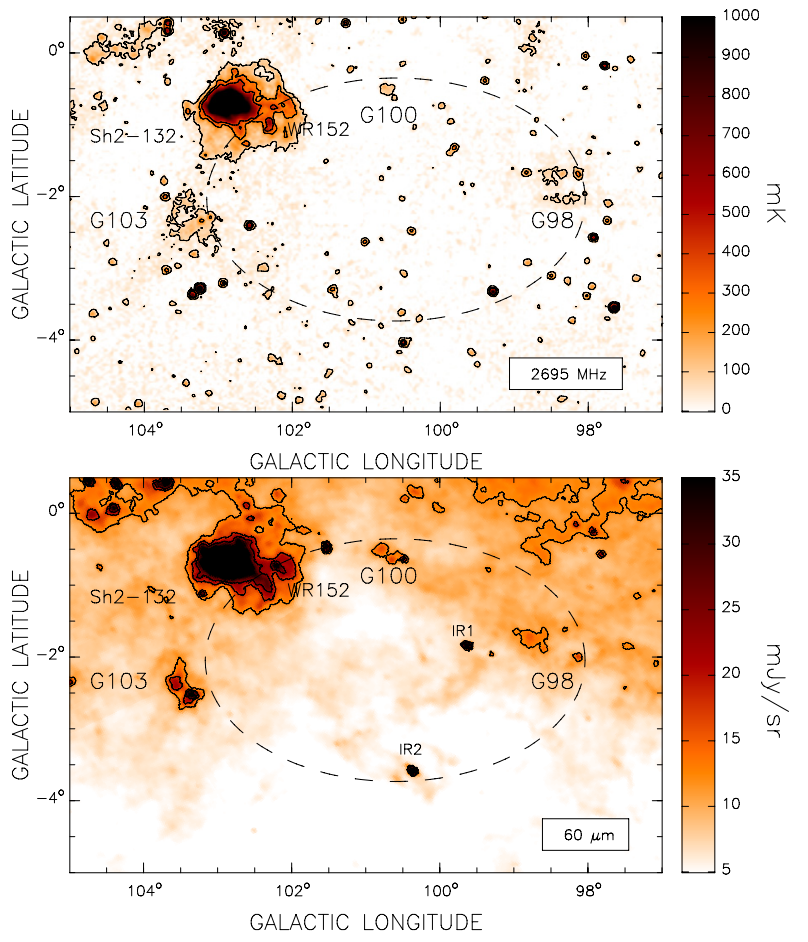

Fig. 6. Upper panel. Effelsberg $2695 \mathrm{MHz}$ emission distribution in the area of GS 100-02-41. Contour levels are at 65, 250 and $400 \mathrm{mK}$. Lower panel. IRIS $60 \mu \mathrm{n}$ emission distribution. Contour levels are at 12,18 and $24 \mathrm{mJy} \mathrm{sr}^{-1}$. In both panels the ellipse marks the location of the large $\mathrm{H}_{\mathrm{I}}$ shell.

panel) toward the region where GS $100-02-41$ is detected. No large-scale features that could be interpreted as the counterpart of the $\mathrm{H}$ I structure could be found in the radio continuum or in the infrared images. The strong source observed at both frequencies at $(l, b) \simeq(102.8,-0.7)$ is the well-known $\mathrm{H}$ II region Sh2-132, which has been studied recently by Vasquez et al. (2010). Toward the southwest of this $\mathrm{H}_{\text {II }}$ region, a ring nebula related to the Wolf-Rayet star WR 152 is also observed (Cappa et al. 2010). On the other hand, three slightly extended and much more weaker sources are seen projected onto the $\mathrm{H}$ i shell. Following the standard nomenclature, they will be referred to as G103.39-2.28 (G103, for short), G100.7-0.5 (G100), and G98.51-1.7 (G98). These three sources will be analyzed in Sect. 4.
In the infrared image (see Fig. 6 lower panel) we observed two sources, IRAS 22036+5306 (IR1) and IRAS $22142+5206(\operatorname{IR} 2)$, located at $(l, b)=\left(99^{\circ} .63,-1.85\right)$ and $(l, b)=$ $(100.38,-3.58)$, respectively. Neither of these sources has a counterpart at $2695 \mathrm{MHz}$. The first source is associated with a post AGB star, whilst the second one (IRAS 22142+5206) may be a protostellar object according to Dobashi et al. (1998). They found IRAS $22142+5206$ to be associated with CO molecular emission at $V=-37.2 \mathrm{~km} \mathrm{~s}^{-1}$.

\subsection{Large $H_{1}$ shell origin}

The origin of GS 100-02-41 may be attributed to the action of the stellar wind of several massive stars and their subsequent supernova explosions. In the following, we analyze both scenarios based on the derived parameters of the shell.

An inspection of the Galactic $O B$ Associations in the Northern Milky Way Galaxy presented by Garmany \& Stencel (1992) reveals that several stars belonging to Cep OB1 are seen projected inside the ellipse delineating GS 100-02-41. They are indicated by symbols in Fig. 2.

Garmany \& Stencel (1992) determined for Cep OB1 a photometric distance modulus of $\mathrm{DM}=12.2 \mathrm{mag}$, which yields a distance of $2.75 \mathrm{kpc}$. On the other hand, based on the stellar proper motions given by Hipparcos, Mel'Nik \& Dambis (2009) estimated a distance of $2.8 \mathrm{kpc}$ for this OB association. This distance is consistent with the distance estimate derived for GS 100-02-41 when non-circular motions are considered (Brand \& Blitz 1993). To test the possibility that the Cep OB1 stars lying inside the $\mathrm{H}_{\mathrm{I}}$ shell could have been capable of creating it, an evaluation of the energy that may be injected into the ISM by the early type stars of Cep OB1 is needed. Is the energy provided by these massive stars sufficient to create GS 100-02-41?

Those stars lying within the boundaries of GS 100-02-41, twenty in total, are listed in Table 3. Column 1 gives the star's identification, Cols. 2 and 3 their galactic coordinates, and Col. 4 their spectral types as given by Garmany \& Stencel (1992). Based on the evolutionary track models published by Schaller et al. (1992), and adopting the bolometric magnitudes and effective temperatures given by Garmany \& Stencel (1992), we estimated the main sequence (MS) spectral type for each star (Col. 5). Column 6 gives an estimate of the star MS lifetime $(t(\mathrm{MS}))$ as derived from the stellar models of Schaller et al. (1992). The values given in this column are a rough estimate as a consequence of the uncertainty in the mass adopted for each star. Columns 7 and 8 give the mass loss rates $(\dot{M})$ and wind velocities $\left(V_{\mathrm{w}}\right)$ taken from Leitherer (1998), respectively. Column 9 gives the total wind energy released by each star during its mainsequence phase, $E_{\mathrm{w}}=0.5 \dot{M} V_{\mathrm{w}}^{2} t(\mathrm{MS})$. Given that HDE 235673 and HDE 235825 are still in the MS, their values are upper limits.

Theoretical models predict that only $20 \%$ of the wind energy is converted into mechanical energy of the shell (Weaver et al. 1977). In the case of GS 100-02-41, this implies that for the total kinetic energy stored in the shell, $E_{\mathrm{k}}=(1.8 \pm 0.8) \times 10^{50} \mathrm{erg}$, a wind energy greater than $9 \times 10^{50}$ erg would be required. However, the analysis of several observed $\mathrm{H}_{\mathrm{I}}$ shells shows that the energy conversion efficiency seems to be lower, roughly about 2-5\% (Cappa et al. 2003).

From the last column of Table 3 we infer that the total wind energy injected during the MS phase of the Cep OB1 stars is $\sim 29.7 \times 10^{50} \mathrm{erg}$, which is sufficient to create GS 100-02-41 if the energy conversion efficiency were $\sim 6 \%$. 
Table 3. Cep OB1 stars lying inside GS 100-02-41.

\begin{tabular}{|c|c|c|c|c|c|c|c|c|}
\hline Star & $l$ & $b$ & Sp. Type & MS Sp. Type & MS lifetime (Myr) & $\log \left(\dot{M}\left(M_{\odot} \mathrm{yr}^{-1}\right)\right)$ & $V_{\mathrm{w}}\left(\mathrm{km} \mathrm{s}^{-1}\right)$ & $E_{\mathrm{w}}\left(10^{50} \mathrm{erg}\right)$ \\
\hline HDE 235673 & $98^{\circ} .4$ & -1.6 & $06.5 \mathrm{~V}$ & 06.5 & 5.6 & -6.27 & 2700 & $\leq 2.2$ \\
\hline HD 209678 & $99: 3$ & -1.8 & B2 I & B0 & 12 & -7.17 & 1600 & 0.2 \\
\hline HD 209900 & 99.7 & -1.7 & A0 Ib & B1 & 26 & -8.2 & 2500 & 0.2 \\
\hline HD 210809 & 99.9 & -3.1 & O9 Iab & $\mathrm{O} 7$ & 6.4 & -6.39 & 2700 & 2.1 \\
\hline $\mathrm{BD}+513135$ & 100.6 & -2.1 & B3 II & B 0.5 & 14 & -7.3 & 1850 & 0.2 \\
\hline HDE 235781 & $101^{\circ} 2$ & -2.6 & B6 Ib & B1 & 22 & -8.2 & 2500 & 0.2 \\
\hline $\mathrm{BD}+532820$ & $101^{\circ} 2$ & -1.7 & B0 IV & B0 & 16 & -7.17 & 1600 & 0.2 \\
\hline $\mathrm{BD}+522833$ & $101^{\circ} .4$ & -2.1 & B1 III & O9.5 & 10 & -7.04 & 2500 & 0.5 \\
\hline $\mathrm{BD}+532837$ & 101.5 & -2.1 & B2 III & B 0.5 & 14 & -7.3 & 1850 & 0.2 \\
\hline HDE 235783 & 101.7 & -1.9 & B1 Ib & B 0.5 & 14 & -7.3 & 1850 & 0.2 \\
\hline $\mathrm{BD}+532843$ & 101.8 & -2.2 & O8 III & $\mathrm{O} 7$ & 6.4 & -6.39 & 2700 & 2.1 \\
\hline $\mathrm{BD}+542718$ & 102.0 & -0.9 & B2 III & B 0.5 & 14 & -7.3 & 1850 & 0.2 \\
\hline $\mathrm{BD}+542726$ & 102.2 & -1.0 & B1.5 II & B 0.5 & 14 & -7.3 & 1850 & 0.2 \\
\hline HDE 235813 & 102.4 & -2.0 & B0 III & O6 & 6.3 & -6.14 & 2700 & 2.6 \\
\hline HILTNER 1106 & 102.5 & -0.7 & B0 III & O8 & 6.9 & -6.65 & 2600 & 0.9 \\
\hline $\mathrm{BD}+532885$ & 102.7 & -2.9 & B2 III & B 0.5 & 14 & -7.3 & 1850 & 0.2 \\
\hline HD 212455 & 102.8 & -1.7 & B6 Ib & B 0.5 & 14 & -7.3 & 1850 & 0.2 \\
\hline HDE 235825 & 102.9 & -1.8 & O9 V & O9 & 8 & -6.91 & 2500 & $\leq 0.6$ \\
\hline $\mathrm{BD}+542764$ & $103^{\circ} 0$ & -1.7 & $\mathrm{~B} 1 \mathrm{Ib}$ & B0.5 & 14 & -7.3 & 1850 & 0.2 \\
\hline $\mathrm{BD}+542761$ & $103^{\circ} .1$ & -1.4 & O6 III & O3* & 4.3 & -5.43 & 3200 & $\leq 16.3$ \\
\hline
\end{tabular}

References. * The bolometric magnitude and effective temperature given by Garmany \& Stencel (1992) lie outside the evolutionary tracks of Schaller et al. (1992). We adopted the earliest spectral type for this star.

It is important to mention that the MS lifetime of all O stars, which are the main energy contributors, is compatible, within errors, with the dynamical age of the shell $(5.5 \pm 2.7 \mathrm{Myr})$. Moreover, the fact that most of the massive stars located inside the shell are evolved stars may explain the absence of ionized gas related to GS 100-02-41.

On the other hand, taking into account that the $\mathrm{SN}$ rate in a typical OB association is about one per $10^{5}-3 \times 10^{5} \mathrm{yr}$ (McCray \& Kafatos 1987), over the lifetime of GS 100-02-41 is reasonable to assume that the most massive members of Cep OB1 may have exploded as an SN. In this case, the energy of the explosions, as well as the energy injected by the stellar winds of the $\mathrm{SNe}$ progenitors, would also have contributed to the formation of the shell. There is no detection of an SNR in either radio or $\mathrm{X}$-ray surveys, but this could be because of the relatively high age of GS 100-02-41. However, an evidence that an SN explosion could have taken place in the region is the presence of the pulsar PSR J2150+5247, located at $(l, b)=(97.5,-0.9)$ (Taylor et al. 1993). Even though the estimated distance to the pulsar is $5.48 \mathrm{kpc}$ (Taylor et al. 1993), bearing in mind that this distance is obtained based on the dispersion measure and a galactic electron density model (Taylor \& Cordes 1993), the error is large, and therefore the possibility for the pulsar to be located at a distance similar to the one of GS 100-02-41 cannot be ruled out. Were this the case, an upper age limit for the progenitor of PSR J2150+5247, assuming it was an early B-type star, would be $\sim 3 \times 10^{7} \mathrm{yr}$, which reasonably agrees with the age of the oldest star, HD 209900, belonging to Cep OB1.

\section{Sources G98, G100, and G103}

\subsection{Their nature}

As mentioned in Sect. 3.2, three slightly extended sources, labeled G98, G100, and G103, are observed at radio continuum and infrared wavelengths, projected onto the border of GS 10002-41. The upper panels of Fig. 7 show the CGPS $1420 \mathrm{MHz}$ radio continuum image of each source. In these images, discrete sources have been removed. The middle panels show the HIRES $60 \mu \mathrm{m}$ emission distribution in the area of G98, G100, and G103. At both frequencies, the original images were smoothed to a 2-arcmin resolution to increase the signal-to-noise ratio.

A clue on their physical nature (thermal or non-thermal) may be provided by the behavior of the flux density as a function of frequency. Previous flux density estimations for G100 were obtained by White \& Becker (1992) and Kerton et al. (2007). White \& Becker (1992) obtained a flux density at $1420 \mathrm{MHz}$ of $456 \mathrm{mJy}$, while Kerton et al. (2007) estimated a lower flux density, $S_{1420}=48.8 \pm 24.0 \mathrm{mJy}$. To estimate the spectral index $\alpha$ $\left(S_{v} \propto v^{-\alpha}\right)$ of each source, CGPS continuum observations at 408 and $1420 \mathrm{MHz}$, and Effelsberg continuum data at $2695 \mathrm{MHz}$ were used in this work.

Flux density determinations, specially for weak sources like the ones we are dealing with, are strongly dependent on the source angular extent, which in turn depends on the outermost reliable continuum level. Before defining the angular extent of each source, the overall galactic continuum emission has to be subtracted. To this end we applied the "background filtering" method (BGF) developed by Sofue \& Reich (1979). A $1^{\circ} \times 1^{\circ}$ filtering beam was applied to all sources at each frequency.

The derived flux densities are given in Table 4. The quoted errors stem from the uncertainty in determining the background levels. The spectral index $\alpha$ was derived from a fit to the observed flux densities, under the assumption that none of these sources is optically thick at the lowest frequency $(408 \mathrm{MHz}$ ). For G100 a residual grating ring from the strong nearby Cas A source falls on top of the source, making the estimate of the $408 \mathrm{MHz}$ flux density meaningless. The lower flux density obtained by Kerton et al. (2007) is consistent with the smaller area considered for G100 by these authors. On the other hand, the higher value given by White \& Becker (1992) may be attributed to the fact that they used Green Bank data (HPBW $\sim 11^{\prime}$ ).

Though highly uncertain, the spectral indices are consistent with a thermal nature for G98, G100, and G103; implying that they are $\mathrm{H}_{\text {II }}$ regions. Another argument in favor of this interpretation comes from the far-infrared data. From the measured flux 

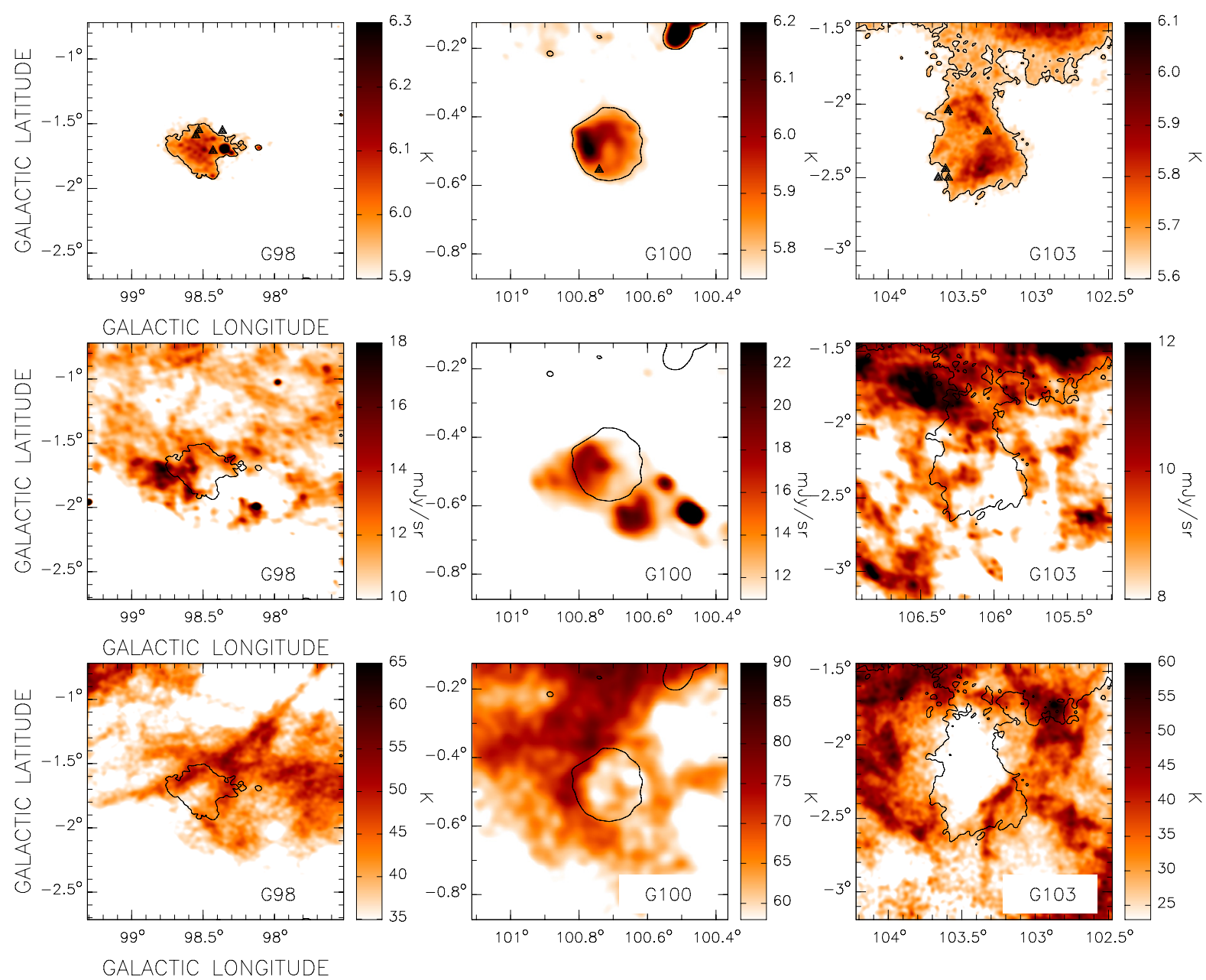

Fig. 7. Upper panels: $1420 \mathrm{MHz}$ radio continuum images in the area of G98 (left), G100 (middle), and G103 (right). Point-like sources have been removed. Contour levels are at 6.0, 5.8, and 5.6 K, for G98, G100, and G103, respectively. The black triangle symbols indicate the location of the stars HD 235673, ALS 12073, ALS 12071 and ALS 12074 (G98), BD+542684 (G100), and ALS 12434, ALS 12443, ALS 12471, ALS 12469 and ALS 12475 (G103). Middle panels: HIRES $60 \mu \mathrm{m}$ emission distribution in the area of G98, G100 and G103. For comparison with the $1420 \mathrm{MHz}$ emission, contour levels are the same as in upper panels. Lower panels: CGPS H i emission distribution averaged in the velocity range from -41.9 to $-50.9 \mathrm{~km} \mathrm{~s}^{-1}$ in the area of G98 (left), from -50.9 to $-55.9 \mathrm{~km} \mathrm{~s}^{-1}$ in the area of G100 (middle) and from -29.5 to $-31.15 \mathrm{~km} \mathrm{~s} \mathrm{~s}^{-1}$ in the area of G103 (right). Contour levels are the same as for upper and middle panels. In all images the angular resolution is 2-arcmin.

Table 4. G98, G100 and G103 parameters.

\begin{tabular}{lccc}
\hline \hline Source & G98 & G100 & G103 \\
\hline Longitude & 98.5 & 100.7 & $103^{\circ} .3$ \\
Latitude & -1.6 & -0.5 & -2.4 \\
Size (arcmin) & $73 \times 64$ & $19 \times 23$ & $54 \times 87$ \\
$S_{408}($ Jy) & $2.1 \pm 0.3$ & - & $2.0 \pm 0.3$ \\
$S_{1420}(\mathrm{Jy})$ & $2.0 \pm 0.1$ & $0.21 \pm 0.03$ & $1.50 \pm 0.15$ \\
$S_{2695}(\mathrm{Jy})$ & $1.9 \pm 0.3$ & $0.19 \pm 0.03$ & $1.70 \pm 0.35$ \\
Spectral index $\alpha$ & $-0.05 \pm 0.01$ & $-0.16 \pm 0.33$ & $-0.11 \pm 0.08$ \\
$S_{60}($ Jy) & $410 \pm 20$ & $120 \pm 6$ & $1030 \pm 50$ \\
$S_{100}(\mathrm{Jy})$ & $1640 \pm 80$ & $380 \pm 20$ & $1840 \pm 90$ \\
Dust temperature (K) & $24.5 \pm 0.7$ & $26.5 \pm 0.8$ & $33.1 \pm 1.1$ \\
\hline
\end{tabular}

densities at 60 and $100 \mu \mathrm{m}$ and following the procedure descibed by Cichowolski et al. (2001), the dust temperature was estimated for each region (see Table 4). The derived temperatures are typical of $\mathrm{H}_{\text {II }}$ regions. The errors quoted for the dust temperatures are formal errors from the fitting procedure.

\subsection{Location of $\mathrm{G} 98, \mathrm{G} 100$ and $\mathrm{G} 103$}

We now try to discern whether these ionized regions could be physically related to GS $100-02-41$. Unfortunately, the radio continuum flux densities of G98, G100 and G103 are not strong enough to allow us to derive a reasonable $\mathrm{H}_{\mathrm{I}}$ absorption spectrum in order to attempt to set a limit to their distances. Another way to attempt to estimate their distances is to look for signatures of the interaction among the ionized regions and the $\mathrm{HI}$ and $\mathrm{CO}$ gas emission observed around these sources. If the continuum sources were related to GS 100-02-41, the $\mathrm{H}$ II regions would expand within the gas of GS 100-02-41 and we would be able to observe for each source an $\mathrm{H}_{\mathrm{I}}$ minimum in a velocity range compatible with the radial velocity of the large $\mathrm{H}_{\mathrm{I}}$ shell. After a thorough inspection of the CGPS H I data cube, we were able to pinpoint $\mathrm{H}_{\mathrm{I}}$ minima that have a good morphological correlation with the radio continuum sources.

Mean brightness temperature of these structures are shown in the lower panels of Fig. 7 (the original images were smoothed to a 2 -arcmin resolution). The radial velocity ranges where these minima are detected range from -40.2 to $-51.8 \mathrm{~km} \mathrm{~s}^{-1}$ 
Table 5. OB stars probably related to G98, G100, and G103.

\begin{tabular}{lccccc}
\hline \hline Star & Galactic coordinates $(l, b)$ & Sp. Type & $v(\mathrm{mag})$ & $M_{v}{ }^{a}$ & Sp. Type $^{a}$ \\
\hline G98 & & & & & \\
HD 235673 & $98.36,-1.55$ & O7 & 9.14 & $-4.7 \pm 0.5$ & O5/O8.5 \\
ALS 12073 & $98.43,-1.71$ & OB & 12.1 & $-1.7 \pm 0.5$ & B2/B5 \\
ALS 12071 & $98.53,-1.55$ & OB & 12.25 & $-1.6 \pm 0.5$ & B2/B5 \\
ALS 12074 & $98.55,-1.59$ & OB & 12.04 & $-1.8 \pm 0.5$ & B2/B5 \\
G100 & & & & & \\
BD +542684 & $100.74,-0.55$ & OB & 10.8 & $-3.0 \pm 0.5$ & B0/B2 \\
G103 & & & & & \\
ALS 12434 & $103^{\circ} .32,-2.18$ & B5 & 10.6 & $-3.2 \pm 0.5$ & B0/B2 \\
ALS 12443 & $103^{\circ} .59,-2.04$ & OB & 12.6 & $-1.2 \pm 0.5$ & B3/B7 \\
ALS 12471 & $103^{\circ} .59,-2^{\circ} .50$ & OB & 12.4 & $-1.4 \pm 0.5$ & B2/B7 \\
ALS 12469 & $103.61,-2.44$ & B2 & 10.6 & $-3.3 \pm 0.5$ & B0/B2 \\
ALS 12475 & $103^{\circ} .66,-2.50$ & B2 & 11.4 & $-2.4 \pm 0.5$ & B1/B3 \\
\hline
\end{tabular}

References. ${ }^{(a)}$ For the adopted distance of $2.8 \pm 0.6$ kpc. ${ }^{(b)}$ Star found in both catalogs and member of Cep OB1 (Garmany \& Stencel 1992$)$.

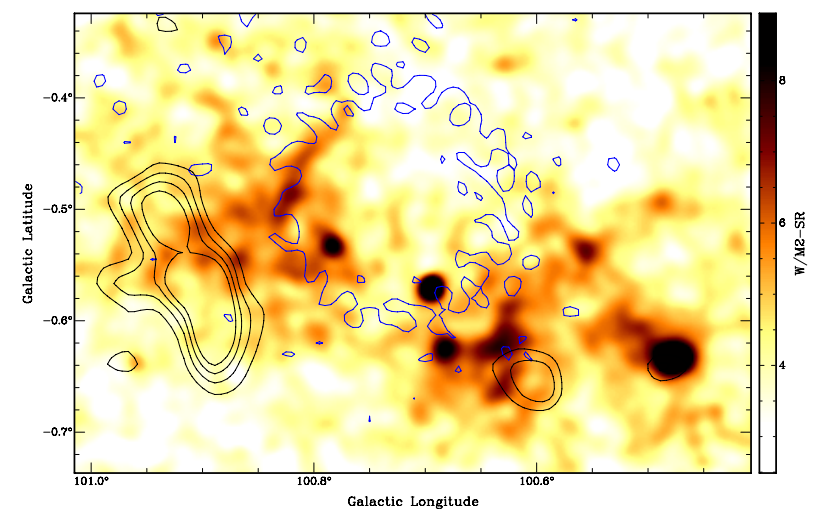

Fig. 8. 8.3 $\mu \mathrm{m}$ emission distribution in the area of G100. The blue contour corresponds to the $5.8 \mathrm{~K}$ level at $1420 \mathrm{MHz}$. Black contours are associated with the $\mathrm{CO}$ emission distribution averaged between -52.25 and $-55.17 \mathrm{~km} \mathrm{~s}^{-1}$, at $0.4,0.6,0.8$ and $1.0 \mathrm{~K}$.

(G98), from -50.1 to $-58.4 \mathrm{~km} \mathrm{~s}^{-1}$ (G100), and from -22.1 to $-33.6 \mathrm{~km} \mathrm{~s}^{-1}$ (G103). However, it is important to mention that for the averages shown in Fig. 7 only those velocity channels were considered where the minima are best defined. To facilitate the comparison among the radio continuum and the $\mathrm{H}_{\mathrm{I}}$ emission distributions, the $1420 \mathrm{MHz}$ contour line defining the source's extent is superimposed on the $\mathrm{H}_{\text {I }}$ images. The $\mathrm{H}_{\text {II }}$ regions are clearly surrounded by enhanced $\mathrm{H}_{\mathrm{I}}$ emission. The positional coincidence between the $\mathrm{H}_{\mathrm{I}}$ features and their corresponding $\mathrm{H}$ II region suggests that they are physically related. It is worth mentioning that although the entire velocity cube was searched for $\mathrm{HI}_{\mathrm{I}}$ features likely to be related to the ionized regions, no other peculiar structures but those shown in Fig. 7 were found.

We have also analyzed the $\mathrm{CO}$ emission distribution in the area of G100 and G103 (unfortunately, there are no CO data available for G98). We found two molecular clouds probably related to $\mathrm{G} 100$ in the velocity range form -52.25 to $-55.17 \mathrm{~km} \mathrm{~s}^{-1}$ (black contours in Fig. 8), in coincidence with the velocity range where the $\mathrm{H}_{\mathrm{I}}$ structure was found. Figure 8 also shows the MSX Band A $(8.28 \mu \mathrm{m})$ image (red colors) and the $5.8 \mathrm{~K}$ level at $1420 \mathrm{MHz}$ (blue contour). Clearly, the $8.28 \mu \mathrm{m}$ emission partially borders the ionized gas. It is worth mentioning that the emission observed in the MSX Band A is not detected in the other three MSX bands $(12.13 \mu \mathrm{m}, 14.65 \mu \mathrm{m}$, and $21.3 \mu \mathrm{m}$ ), suggesting that the polycyclic aromatic hydrocarbons (PAHs) could be mainly responsible for the emission detected at
$8.28 \mu \mathrm{m}$, indicating the presence of a photo-dissociated region (PDR) in the border of G100. An inspection of the CO data cube in the area of G103 did not reveal any CO structure that could probably be related to the $\mathrm{H}$ II region.

Bearing in mind that GS 100-02-41 is detected in the velocity range from -29 to $-51.7 \mathrm{~km} \mathrm{~s}^{-1}$ and that its baricentral velocity is $V_{0}=-41 \pm 2 \mathrm{~km} \mathrm{~s}^{-1}$, we would locate both G98 and G100 in the approaching hemisphere of GS 100-02-41, whilst G103 would be placed in its receding part. Below we adopt the GS 100-02-41 distance for the H II regions.

\subsection{Exciting stars of G98, G100, and G103}

To identify the exciting stars of G98, G100, and G103 we have inspected the Galactic O Star Catalog (Sota et al. 2008) and the OB Star Catalog (Reed 2003) looking for massive stars located in projection toward the $\mathrm{H}$ II regions.

Only one star projected within the border of G98 is listed by Sota et al. (2008), whilst in the Reed catalog four stars are seen projected toward G98 (one of them coincides with the one catalogued by Sota et al. 2008), one onto G100, and five onto G103. They are listed in Table 5 and their positions are indicated by triangle symbols in Fig. 7. It is worth mentioning that HD 235673, the star seen projected onto G98, appears in Table 3 as a Cep OB1 member and therefore was considered as an input energy source for the creation of GS 100-02-41. Nonetheless, its contribution to the overall energy budget is $\sim 7 \%$, so if this star were related to G98, its exclusion as being responsible in the creation of GS 100-02-41 would not modify the conclusions reached in Sect. 3.3.

To check whether these stars may have contributed to the creation of the observed structures, under the assumption that they are located at the same distance than the $\mathrm{H}_{\text {II }}$ regions $(2.8 \pm$ $0.6 \mathrm{kpc}$ ), we analyzed if their absolute magnitudes are compatible with those corresponding to OB-type stars. We first needed to estimate the visual absorption in the region. From an averaged $\mathrm{H}_{\mathrm{I}}$ profile (from 0 to $-41 \mathrm{~km} \mathrm{~s}^{-1}$ ) of the whole solid angle covered by GS $100-02-41$, we derive a total $\mathrm{H}_{\mathrm{I}}$ column density of about $N_{\mathrm{HI}}=3 \times 10^{21} \mathrm{~cm}^{-2}$. Using the relation $A_{v}=5.3 \times 10^{-22} N_{\mathrm{H} \text { I }}$ (Bohlin et al. 1978), we estimate $A_{v}=1.6 \mathrm{mag}$. As a check on this, for HD 235673 we estimated the visual absorption using $A_{v}=3.1 E(B-V)$, where $E(B-V)=(B-V)-(B-V)_{0}$. For $(B-V)_{0}=-0.32$ (Schmidt-Kaler 1982) and $(B-V)=0.21$ (Hiltner \& Johnson 1956) we obtain $A_{v}=1.6$. Both values agree with the absorption 
of 1-2 mag given by Neckel \& Klare (1980). Then, adopting a visual absorption of $1.6 \mathrm{mag}$, the absolute magnitudes (see Col. 5) of the stars listed in Table 5 were calculated. Based on Martins \& Plez (2006) and Schmidt-Kaler (1982), the corresponding spectral types were estimated (see Col. 6 of Table 5).

Given that at the assumed distance all stars listed in Table 5 could be O- or early B-type stars, we suggest that they may be responsible of creating G98, G100, and G103.

Next, we should consider whether the number of UV ionizing photons needed to keep the continuum sources ionized could be provided by their probably exciting stars. The total number of Lyman continuum photons is given by $N_{\mathrm{Lym}}=$ $0.76 \times 10^{47} T_{4}^{-0.45} v_{\mathrm{GHz}}^{0.1} D_{\mathrm{kpc}}^{2} S_{v}$ (Chaisson 1976), where $T_{4}$ is the electron temperature in units of $10^{4} \mathrm{~K}, D_{\mathrm{kpc}}$ is the distance in kpc, $v_{\mathrm{GHz}}$ is the frequency in $\mathrm{GHz}$ and $S_{v}$ is the measure flux density in Jy. Using the $1420 \mathrm{MHz}$ flux densities given in Table 4, a distance of $2.8 \mathrm{kpc}$ and adopting $T_{4}=1$, we obtained $N_{\text {Lym }}=1.2 \times 10^{48} \mathrm{~s}^{-1}$ for G98, $N_{\text {Lym }}=1.3 \times 10^{47} \mathrm{~s}^{-1}$ for G100, and $N_{\text {Lym }}=1.8 \times 10^{48} \mathrm{~s}^{-1}$ for G103. According to the theoretical models of Schaerer \& de Koter (1997), for solar metallicity, the estimated $N_{\text {Lym }}$ necessary to keep G98, G100, and G103 ionized, could be provided by a $09.5 \mathrm{~V}, \mathrm{~B} 0.5 \mathrm{~V}$, and $09.5 \mathrm{~V}$ stars (or two B0V), respectively. This indicates that the joint action of the stars listed in Table 5 can provide the ionizing photons needed for each $\mathrm{H}_{\text {II }}$ region. In the case of G98, given that the contribution of HD 235673 is essential to keep this region ionized, we conclude that this star is associated with G98 instead of being related to the genesis of GS 100-02-41 as was considered in Sect. 3.3. This implies that the wind energy provided for this star should not have been taken into account for the origin of GS 100-02-41. As mentioned before, this does not affect the scenario proposed for the creation of the large H I shell.

\section{Triggered star formation?}

It is generally believed that expanding shells may induce star formation at their edges (Elmegreen 1998). Shells behind shock fronts experience gravitational instabilities that may lead to the formation of high condensations inside the swept-up gas, and some of them may produce new stars. An increasing body of observational evidence supports the importance of the shell's evolution in creating new stars (Patel et al. 1998; Oey et al. 2005; Arnal \& Corti 2007; Cichowolski et al. 2009; Cichowolski \& Pineault 2011).

In our case, having an old large $\mathrm{H}_{\mathrm{I}}$ shell containing several $\mathrm{H}$ II regions in its edge that seems to be at the same distance, we wonder if this could be another case of triggered star formation. Were this the case, we would expect an age gradient in the region, in the sense that G98, G100, and G103 should be younger than GS 100-02-41. Below we attempt to estimate the age of the $\mathrm{H}$ II regions G98, G100, and G103, and compare them with the dynamical age of GS 100-02-41 estimated in Sect. 3.1 (see Table 2).

As a rough estimate to the age of each region, we evaluated their dynamical ages as $t_{\mathrm{dyn}}=0.6 R / V_{\mathrm{e}}$ (Weaver et al. 1977). The radius $R$ of each region was estimated from the sizes given in Table 4. We infered $R=27.8 \pm 6.0,8.5 \pm 1.8$ and $27.9 \pm 6.0 \mathrm{pc}$, for G98, G100 and G103, respectively, where the errors stem from the uncertainty on the distance. Relying on the velocity ranges where the $\mathrm{H}_{\mathrm{I}}$ emission associated with each $\mathrm{H}_{\text {II }}$ region is observed (see Sect. 4), expansion velocities of $5.8 \mathrm{~km} \mathrm{~s}^{-1}$ (G98), $4.2 \mathrm{~km} \mathrm{~s}^{-1}$ (G100) and $5.8 \mathrm{~km} \mathrm{~s}^{-1}$ (G103) were assumed. Thus, we obtain an age of $2.9 \pm 0.9,1.2 \pm 0.5$ and $2.9 \pm 0.9 \mathrm{Myr}$ for
G98, G100, and G103, respectively. The quoted errors stem from the uncertainties in the sizes and from the assumption that the expansion velocities are accurate to within $1.3 \mathrm{~km} \mathrm{~s}^{-1}$ (one velocity channel).

From these estimates, we conclude that G98, G100, and G103 are younger than GS 100-02-41. This age difference is supported by the fact that most of the stars probably responsible for creating GS 100-02-41 have already evolved from the MS, while the ionized gas of the $\mathrm{H}$ II regions is still observed.

As mentioned in Sect. 3.2, in addition to G98, G100, and G103, three interesting sources are seen projected onto the border of GS 100-02-41: the $\mathrm{H}_{\text {II }}$ region Sh2-132, the ring nebula associated with WR 152, and the infrared source IRAS $22142+5206$

Regarding Sh2-132 and the ring nebula associated with WR 152, Vasquez et al. (2010) and Cappa et al. (2010) found neutral gas interacting with the $\mathrm{H}_{\mathrm{II}}$ regions in the velocity ranges from -38 to $-53 \mathrm{~km} \mathrm{~s}^{-1}$ and from -43 to $-52 \mathrm{~km} \mathrm{~s}^{-1}$, respectively. For Sh2-132 this coincides with the ionized velocity gas (Georgelin \& Georgelin 1976; Chu \& Treffers 1981; Reynolds 1988; Fich et al. 1990; Quireza et al. 2006). Taking into account non-circular motions in this part of the Galaxy (Brand \& Blitz 1993), Vasquez et al. (2010) and Cappa et al. (2010) inferred for these regions a kinematical distance of $3.5 \pm 1.0 \mathrm{kpc}$. They argued that this value closely agrees with the distance estimates of the main exciting stars of the regions, WR $153 \mathrm{ab}$ and WR 152. On the other hand, bearing in mind that GS 100-02-41 is observed in the velocity range from -29.0 to $-51.7 \mathrm{~km} \mathrm{~s}^{-1}$, and that the $\mathrm{H}_{\text {II }}$ regions are located at its border, we suggest that both ionized structures may be located at the same distance as GS 100-02-41, and that the velocity differences are because the $\mathrm{H}_{\text {II }}$ regions are located in the approaching part of the large shell. Although Vasquez et al. (2010) do not give any age estimate for Sh2-132, the fact that it is being ionized by WR 153ab implies that Sh2-132 is probably older than the period of time spent by the progenitor of the WR in the main-sequence phase. Given that WR $153 \mathrm{ab}$ is a WN6.5 star, the mass of the progenitor was probably about 40-50 $M_{\odot}$ (Crowther 2007). According to Schaller et al. (1992), the MS lifetime for such a star is about 4 Myr. Concerning the age of the ring nebula associated with WR 152, Cappa et al. (2010) estimated a dynamical age of 1 Myr for the associated wind-blown bubble. However, knowing that according to Cappa et al. (2010), large errors are involved in this estimate and bearing in mind the time that the progenitor of the WR star spent in the MS phase, the age of the $\mathrm{H}_{\text {II }}$ region is probably higher, closer to the one estimated above for Sh2-132.

With respect to IRAS $22142+5206$, as mentioned in Sect. 3.2, this infrared source has a massive molecular outflow associated with it at the velocity of $-37.2 \mathrm{~km} \mathrm{~s}^{-1}$ (Dobashi et al. 1998). The coincidence between this velocity and the velocity range where GS 100-02-41 is observed suggests that they may be located at the same distance. Based on the total luminosity of IRAS 22142+5206, Dobashi et al. (1998) suggest that this source will evolve into a late O-type or an early B-type star.

In summary, the fact that G98, G100, G103, Sh2-132, the ring nebula associated with WR 152, and IRAS 22142+5206 lie at the edge of GS 100-02-41 and seem to be at the same distance, together with the age gradient, suggest that these sources could have been triggered by the expansion of GS 100-02-41.

\section{Conclusions}

The large H I shell GS 100-02-41 has been analyzed to study the interaction of massive stars with the interstellar medium and, 
in particular, the process of triggered star formation. From this analysis we conclude the following:

GS 100-02-41 is a large shell of a radius of about $102 \mathrm{pc}$ located at a distance of $2.8 \pm 0.6 \mathrm{kpc}$. The swept-up mass in the shell is $(1.5 \pm 0.7) \times 10^{5} M_{\odot}$ and the shell density $n_{\mathrm{sh}}=2.5 \pm$ $0.4 \mathrm{~cm}^{-3}$. The shell is expanding at a velocity of $11 \pm 2 \mathrm{~km} \mathrm{~s}^{-1}$ and its kinetic energy is $(1.8 \pm 0.8) \times 10^{50} \mathrm{erg}$.

Several evolved massive stars members of Cep OB1 are projected inside the large shell. The distance to the OB association is compatible with the kinematical distance of GS 100-02-41 when non-circular motions are considered. An energetical analysis suggests that the wind energy provided during the mainsequence phase of the stars could explain the origin of the shell. However, taking into account the $\mathrm{SN}$ rate in OB associations, the energy contribution of an SN explosion as well as of its massive progenitor cannot be discarded.

From the $2695 \mathrm{MHz}$ radio continuum and $60 \mu \mathrm{m}$ infrared images, we found three slightly extended sources, labeled G98, G100, and G103 projected onto the borders of GS 100-02-41. From the radio flux densities estimated at different wavelengths, the thermal nature of the sources was confirmed by the estimation of their spectral indices. In addition, dust temperatures were estimated and found to be typical of $\mathrm{H}_{\text {II }}$ regions.

An inspection of the $1^{\prime}$ CGPS $\mathrm{H}_{\mathrm{I}}$ data reveals $\mathrm{H}_{\mathrm{I}}$ minima that have a good morphological correlation with the $\mathrm{H}$ II regions at velocity ranges compatible with the velocity spanning by GS 100-02-41. This leads to the conclusion that G98, G100, and G103 are located at the same distance as GS 100-02-41.

From $\mathrm{O}$ and $\mathrm{OB}$ star catalogs, the massive star candidates to be responsible for the ionized gas were identified.

The obtained age difference among the $\mathrm{H}_{\text {II }}$ regions and the shell, together with their relative location leads us to the conclusion that G98, G100, and G103 may have been created as a consequence of the action of a strong shock produced by the expansion of GS 100-02-41 into the surrounding gas.

Acknowledgements. The CGPS is a Canadian Project with international partners and is supported by grants from NSERC. Data from the CGPS are publicly available through the facilities of the Canadian Astronomy Data Centre (http:// cadc.hia.nrc.ca) operated by the Herzberg Institute of Astrophysics, NRC. This project was partially financed by the Consejo Nacional de Investigaciones Científicas y Técnicas (CONICET) of Argentina under project PIP 01299, Agencia PICT 00902, UBACyT 20020090200039, and UNLP G091. L.A.S. is a doctoral fellow of CONICET, Argentina. S.C. and M.A. are members of the Carrera del Investigador Científico of CONICET, Argentina. J.C.T. is member of the Carrera del Personal de Apoyo, CONICET, Argentina.

\section{References}

Arnal, E. M., \& Corti, M. 2007, A\&A, 476, 255

Bohlin, R. C., Savage, B. D., \& Drake, J. F. 1978, ApJ, 224, 132

Brand, J., \& Blitz, L. 1993, A\&A, 275, 67

Cappa, C. E., Arnal, E. M., Cichowolski, S., Goss, W. M., \& Pineault, S. 2003, in A Massive Star Odyssey: From Main Sequence to Supernova, ed. K. van der Hucht, A. Herrero, \& C. Esteban, IAU Symp., 212, 596

Cappa, C. E., Vasquez, J., Pineault, S., \& Cichowolski, S. 2010, MNRAS, 403, 387

Cazzolato, F., \& Pineault, S. 2003, AJ, 125, 2050

Chaisson, E. J. 1976, in Frontiers of Astrophysics, ed. E. H. Avrett, 259
Chakraborti, S., \& Ray, A. 2011, ApJ, 728, 24

Chu, Y.-H., \& Treffers, R. R. 1981, ApJ, 250, 615

Cichowolski, S., \& Pineault, S. 2011, A\&A, 525, A121

Cichowolski, S., Pineault, S., Arnal, E. M., et al. 2001, AJ, 122, 1938

Cichowolski, S., Romero, G. A., Ortega, M. E., Cappa, C. E., \& Vasquez, J. 2009, MNRAS, 394, 900

Crowther, P. A. 2007, ARA\&A, 45, 177

Dobashi, K., Yonekura, Y., Hayashi, Y., Sato, F., \& Ogawa, H. 1998, AJ, 115, 777

Ehlerová, S., \& Palouš, J. 2005, A\&A, 437, 101

Elmegreen, B. G. 1998, in Origins, ed. C. E. Woodward, J. M. Shull, \& H. A. Thronson, Jr., ASP Conf. Ser., 148, 150

Fich, M., Dahl, G. P., \& Treffers, R. R. 1990, AJ, 99, 622

Fürst, E., Reich, W., Reich, P., \& Reif, K. 1990, A\&AS, 85, 691

Garmany, C. D., \& Stencel, R. E. 1992, A\&AS, 94, 211

Georgelin, Y. M., \& Georgelin, Y. P. 1976, A\&A, 49, 57

Heiles, C. 1979, ApJ, 229, 533

Heiles, C. 1984, ApJS, 55, 585

Hiltner, W. A., \& Johnson, H. L. 1956, ApJ, 124, 367

Jung, J. H., Koo, B.-C., \& Kang, Y.-H. 1996, AJ, 112, 1625

Kalberla, P. M. W., Burton, W. B., Hartmann, D., et al. 2005, A\&A, 440, 775

Kerton, C. R., Murphy, J., \& Patterson, J. 2007, MNRAS, 379, 289

Könyves, V., Kiss, C., Moór, A., Kiss, Z. T., \& Tóth, L. V. 2007, A\&A, 463, 1227

Landecker, T. L., Dewdney, P. E., Burgess, T. A., et al. 2000, A\&AS, 145, 509

Leitherer, C. 1998, in Stellar astrophysics for the local group: VIII Canary

Islands Winter School of Astrophysics, ed. A. Aparicio, A. Herrero, \& F. Sánchez, 527

Martins, F., \& Plez, B. 2006, A\&A, 457, 637

McClure-Griffiths, N. M., Dickey, J. M., Gaensler, B. M., \& Green, A. J. 2002, ApJ, 578, 176

McCray, R., \& Kafatos, M. 1987, ApJ, 317, 190

Mel'Nik, A. M., \& Dambis, A. K. 2009, MNRAS, 400, 518

Miville-Deschênes, M.-A., \& Lagache, G. 2005, ApJS, 157, 302

Neckel, T., \& Klare, G. 1980, A\&AS, 42, 251

Oey, M. S., Watson, A. M., Kern, K., \& Walth, G. L. 2005, AJ, 129, 393

Patel, N. A., Goldsmith, P. F., Heyer, M. H., Snell, R. L., \& Pratap, P. 1998, ApJ, 507, 241

Perna, R., \& Raymond, J. 2000, ApJ, 539, 706

Pineault, S. 1998, AJ, 115, 2483

Price, S. D., Egan, M. P., Carey, S. J., Mizuno, D. R., \& Kuchar, T. A. 2001, AJ, 121,2819

Quireza, C., Rood, R. T., Balser, D. S., \& Bania, T. M. 2006, ApJS, 165, 338

Reed, B. C. 2003, AJ, 125, 2531

Reich, W., Fuerst, E., Haslam, C. G. T., Steffen, P., \& Reif, K. 1984, A\&AS, 58, 197

Reich, W., Fuerst, E., Reich, P., \& Reif, K. 1990, A\&AS, 85, 633

Reynolds, R. J. 1988, ApJ, 333, 341

Schaerer, D., \& de Koter, A. 1997, A\&A, 322, 598

Schaller, G., Schaerer, D., Meynet, G., \& Maeder, A. 1992, A\&AS, 96, 269

Schmidt-Kaler, T. 1982, in Landolt-Bornstein: Numerical Data and Functional Relationships in Science and Technology, New Series, Vol. 2b, Stars and Star Clusters, ed. K. Schaifus, \& H. H. Vogt

Sofue, Y., \& Reich, W. 1979, A\&AS, 38, 251

Sota, A., Maíz-Apellániz, J., Walborn, N. R., \& Shida, R. Y. 2008, Rev. Mex. Astron. Astrofis. Conf. Ser., 33, 56

Stanimirović, S. 2007, in IAU Symp. 237, ed. B. G. Elmegreen, \& J. Palous, 84

Stil, J. M., \& Irwin, J. A. 2001, ApJ, 563, 816

Taylor, J. H., \& Cordes, J. M. 1993, ApJ, 411, 674

Taylor, J. H., Manchester, R. N., \& Lyne, A. G. 1993, ApJS, 88, 529

Taylor, A. R., Gibson, S. J., Peracaula, M., et al. 2003, AJ, 125, 3145

Tenorio-Tagle, G. 1981, A\&A, 94, 338

Uyaniker, B., \& Kothes, R. 2002, ApJ, 574, 805

Vasquez, J., Cappa, C. E., Pineault, S., \& Duronea, N. U. 2010, MNRAS, 405, 1976

Weaver, R., McCray, R., Castor, J., Shapiro, P., \& Moore, R. 1977, ApJ, 218, 377

White, R. L., \& Becker, R. H. 1992, ApJS, 79, 331 